

Research at the KVI (a joint venture between the University of Groningen and the FOM foundation) which makes use of light and heavy ions in various disciplines will receive a major boost with the installation of the second-generation, compact, superconducting, variable energy cyclotron AGOR. Shown fully assembled in the photograph, AGOR was built at the IPN Orsay and delivery to Groningen to replace the old (1972 construction) Philips cyclotron with a bending ratio $K$ of 160 is now scheduled for April or May following beam testing in Orsay. The $K=600$ machine will offer $200 \mathrm{MeV}$ protons, $400 \mathrm{MeV}$ alpha and $3 \mathrm{He}$ particles, $100 \mathrm{MeV} / \mathrm{u}$ light ions, and heavy ions down to $U$ ions of several $\mathrm{MeV} / \mathrm{u}$ for a multi-disciplinary facility. Nuclear physics will benefit from the higher energies (and polarized proton and deuteron beams) for studying nuclear excitations, nuclei in extreme conditions of temperature and angular momentum, and the influence of the nuclear medium on the nucleon-nucleon interaction. Atomic physics research on the interaction of multiply charged ions with different targets is to profit from the installation of a recoil-ion source that will deliver very slow ions with much higher charges than can be generated with ECR sources. A medical physics facility for proton therapy is also being considered. A Programme Advisory Committee has already been formed and M.H. Harakeh, Deputy-Director and responsible for the experimental scientific programme (the Director is R. Malfliet), emphasizes that external users as well as ideas for experiments in all fields are very welcome.

colliding with a $\mathrm{Ag}$ target. Lines corresponding to transitions from states with various L-shell fillings are clearly identified. The fact that the line at the lowest energy, corresponding to emission from a completely filled L-shell, is relatively strong implies that the filling of the L-shell is faster than its depopulation by radiative or Auger transitions. Auger cascades are unlikely to yield such a fast filling. In general therefore, changes of relative intensities of the various emission lines as a function of collision velocity and geometry can be used to test

\section{EPS on World-Wide Web}

The main information retrieval systems used on the all-pervasive Internet electronic communication network are Gopher, WIAS and WorldWide Web (W3). W3 is the newest and most powerful as it provides novel features based on combining hypertext and information retrieval techniques while offering access to Gopher and WAIS servers using a client-server paradigm. So W3 users can browse with ease through a global web of network-accessible information. Originally aimed at the high-energy physics community (with CERN as the instigator and driving force), W3 has spread into many other fields. While the number of W3 servers is today smaller than the 1000 or so available on Gopher, it is growing rapidly.

W3 consists of nodes (or documents) and links which are the connections between documents. To follow a link a user simply "clicks on" a highlighted word in a source document (or types in a number if a mouse cannot be used). This causes the linked-to document to be displayed. The document may be one of a variety of different media types, or it can be a searchable index of the sort used in Gopher. A Réseaux Associés pour la Recherche Européenne (RARE) report has recommended con- different scenarios for the population and depopulation of the L-shell.

For the case of bare projectile ions, the same type of X-ray experiments can also exploit the sequential emission of two photons, and Briand et al. [8] were in fact the first to apply this technique (they used bare $\mathrm{Ar}^{18^{+}}$ions). The two satellite spectra of sequentially emitted $\mathrm{Ly}_{\alpha}$ photons provide a measure of the L-shell filling at the corresponding times. L-shell filling rates within the solid could thus be monitored on a femtosecond time-scale. For Ar ions, Briand

centrating on W3 because of its flexibility, the availability of links, and the fact that it is an integrated system combining WAIS and Gopher support.

So W3 was the obvious choice for the Society's East-West Coordination Committee (EWCC) in making information available to physical societies and physicists throughout Europe. A W3 server has been set up by Eddy Lingeman with help from colleagues at CERN and at NIKHEF in Amsterdam; it is now running in the experimental mode with information on EPS, short items taken from Europhysics News, and some major documents such as the Directory of Physics Institutes in Central Europe published last year by EPS (available in soft-cover form from the EPS Secretariat; price: SFR 120.-)

To access the EPS information you need to call up your local W3 server with a command such as "www". A screen is displayed on which you select "high energy physics labs" with your mouse or by typing a number. Up comes another screen with a list of the labs. You select "NIKHEF" and on the NIKHEF screen you will find "EPS". Click it and you will see the EPS

- Client-browser terminal software for fetching and displaying data is available for many sysscreen. Some details as follows: et al. found that four additional electrons on average had entered the L-shell within the 20 fs which elapse between the two sequential Ly $\mathrm{L}_{\alpha}$ transitions.

\section{Conclusions}

The combined information on the collision of highly charged ions with surfaces, obtained from analyses of scattered ions as well as of ejected electrons and photons, has led to a detailed understanding of the electronic processes which take place near the surface. It is now clear that although the projectiles are neutralized at relatively large distances in front of the surface, they carry most of their potential energy into the surface layers of the solid. Surface modifications by highly charged ions via Coulomb explosion processes, for example, are therefore likely to occur. Indeed, recent experiments by our group involving the collision of multiply charged ions with $\mathrm{Si}$ surfaces demonstrate that for sufficiently low kinetic energies $(\approx 2 \mathrm{keV})$, the yield of ejected $\mathrm{Si}$ ions increases dramatically with increasing projectile ions (and with decreasing kinetic projectile energy). Intensive studies of the phenomena involved are foreseen for the future in view of possible technological applications for fabricating surface structures on a nanometre scale.

[1] Winter H., Europhys. Lett. 18 (1992) 207. [2] Burgdörfer J., Lerner P. \& Meyer F.W., Phys. Rev. A 44 (1991) 5674

[3] De Zwart S.T., Drentje A.G., Boers A.L. \& Morgenstern R., Surf. Sci. 217 (1989) 298; Das J. \& Morgenstern R., Phys. Rev. A 47 (1993) 755. [4] Meyer F.W., et al., Phys. Rev. A 44 (1991) 7214; Köhrbrück R., et al., Phys. Rev. A 45 (1992) 4653; Andrä H.J., et al., Suppl. Z. Phys. D 21 (1991) $\mathrm{S} 135$.

[5] Kurz H., et al., Phys. Rev. Lett. 69 (1992) 1140.

[6] Das J., Folkerts L. \& Morgenstern R., Phys. Rev. A 45 (1992) 4669

[7] Briand J.P., et al., Phys. Rev. Lett. 65 (1990) 159.

[8] Briand J.P., et al., Phys. Rev. A 43 (1991) 565.

tems including Macintosh, X/Motif, PC/MS Windows and NEXT; sever software is available for VM mainframes, UNIX, Macintosh, and VMS.

- All W3 compatible programs must be capable of handling the HyperText Markup Language as HTML provides one, simple format for providing linked information. W3 uses Internet's Hypertext Transfer Protocol (HTTP) to allow a browser program to request a keyword search by a remote user (i.e., to allow transfer representations to be negotiated between client and server).

- The W3 addressing mechanism means that an interface to Gopher, WIAS and anonymous FTP information sources may be established in a transparent way.

\section{For further information:}

- Telenet to "info.cern.ch" with user name "www" (no password): then follow instructions for the line-mode browser.

- The 1st International Conference on the Worid-Wide Web will be held at CERN on 2527 May 1994. Contact: Anne Perrelle, CERN, Geneva (tel.: +41-22-793-2406; fax: +41-22793-8730; email: www94@www1.cern.ch) or telnet to "www94.cern.ch" with logon "www94" (no password). 